\title{
Drug-induced anaphylaxis in China: a 10 year retrospective analysis of the Beijing Pharmacovigilance Database
}

\author{
Ying Zhao ${ }^{1,2,3} \cdot$ Shusen $\mathrm{Sun}^{4} \cdot$ Xiaotong $\mathrm{Li}^{1,3} \cdot \mathrm{Xiang} \mathrm{Ma}^{1} \cdot$ Huilin Tang ${ }^{5} \cdot$ Lulu Sun $^{2} \cdot$ \\ Suodi Zhai ${ }^{1} \cdot$ Tiansheng Wang ${ }^{1,3,6}$
}

Received: 9 May 2017 / Accepted: 19 September 2017 / Published online: 31 October 2017

(C) The Author(s) 2017. This article is an open access publication

\begin{abstract}
Background Few studies on the causes of drug-induced anaphylaxis (DIA) in the hospital setting are available. Objective We aimed to use the Beijing Pharmacovigilance Database (BPD) to identify the causes of DIA in Beijing, China. Setting Anaphylactic case reports from the BPD provided by the Beijing Center for Adverse Drug Reaction Monitoring. Method DIA cases collected by the BPD from January 2004 to December 2014 were adjudicated. Cases were analyzed for demographics, causative drugs and route of administration, and clinical signs and outcomes. Main outcome measure Drugs implicated in DIAs were identified and the signs and symptoms of the DIA cases were analyzed. Results A total of 1189 DIA cases were analyzed. The mean age was 47.6 years, and $732(61.6 \%)$ were aged from 18 to 59 years. A total of 627 patients (52.7\%) were females. There was a predominance of cardiovascular (83.8\%) followed by respiratory (55.4\%), central nervous
\end{abstract}

Electronic supplementary material The online version of this article (doi:10.1007/s11096-017-0535-2) contains supplementary material, which is available to authorized users.

Ying Zhao and Shusen Sun have contributed equally to this work.

Suodi Zhai

zhaisuodi@163.com

$\triangle$ Tiansheng Wang

tianwang@unc.edu

1 Department of Pharmacy, Peking University Third Hospital, Beijing, China

2 Department of Pharmacy, Beijing Shijitan Hospital, Beijing, China

3 Department of Pharmacy Administration and Clinical Pharmacy, School of Pharmaceutical Sciences, Peking University, Beijing, China
(50.1\%), mucocutaneous (47.4\%), and gastrointestinal symptoms (31.3\%). A total of 249 different drugs were involved. DIAs were mainly caused by antibiotics (39.3\%), traditional Chinese medicines (TCM) (11.9\%), radiocontrast agents (11.9\%), and antineoplastic agents (10.3\%). Cephalosporins accounted for majority (34.5\%) of antibiotic-induced anaphylaxis, followed by fluoroquinolones $(29.6 \%)$, betalactam/beta-lactamase inhibitors (15.4\%) and penicillins $(7.9 \%)$. Blood products and biological agents $(3.1 \%)$, and plasma substitutes $(2.1 \%)$ were also important contributors to DIAs. Conclusion A variety of drug classes were implicated in DIAs. Patients should be closely monitored for signs and symptoms of anaphylaxis when medications are administered especially with antibiotics, TCM, radiocontrast and antineoplastic agents.

Keywords China $\cdot$ Clinical features $\cdot$ Drug category · Drug-induced anaphylaxis $\cdot$ Pharmacovigilance

4 College of Pharmacy and Health Sciences, Western New England University, Springfield, Massachusetts, USA

5 Department of Epidemiology, Richard M. Fairbanks School of Public Health, Indiana University-Purdue University Indianapolis, Indianapolis, IN, USA

6 Department of Epidemiology, University of North Carolina at Chapel Hill, Chapel Hill, North Carolina, USA 


\section{Impacts on practice}

- Drug-induced anaphylaxis accounts for at least $12 \%$ of adverse drug event reports in Chinese hospitals collected by the Beijing Pharmacovigilance Database between 2004 to 2014 , and the associated mortality rate is 3.3\%.

- The top four drug categories implicated in drug induced anaphylaxis cases in China are antibiotics, traditional Chinese medicines, radiocontrast media and antineoplastic agents.

- Drug induced anaphylaxis most often presents with cardiovascular system symptoms, followed by mucocutaneous, respiratory and central nervous system symptoms.

\section{Introduction}

Anaphylaxis is a severe, life-threatening, systemic allergic reaction that occurs rapidly after contact with an inducing substance. Common triggers of anaphylaxis include food, insect stings, drugs and latex [1,2]. Susceptibility (or incidence) of anaphylaxis varies with age, allergen exposure, and predisposing genetic factors [1,3]. Symptoms of anaphylaxis may progress rapidly and involve multiple target organ systems including the integumentary, respiratory, gastrointestinal, and cardiovascular systems [1].

Of the most common triggers of anaphylaxis [1,2], drugs are considered to be the primary triggers in adults $[1,4]$. Administration of any drug by any route can potentially cause anaphylaxis $[2,5]$. According to a retrospective U.S. epidemiology study, medications were the most common cause $(58.8 \%)$ of 2458 anaphylaxis-related deaths from 1999 to 2010 [6]. Furthermore, a multicenter retrospective study from Korea, drug-induced anaphylaxis (DIA) accounted for $46.5 \%$ of all 1806 anaphylaxis cases, becoming the most common trigger of anaphylaxis in Korea [7].

Although the epidemiological data of DIA have been reported in western countries $[6,8,9]$, data is limited in Asian population. Most published studies are case reports or case series focused on specific drugs such as antibiotics or special clinical situations for instance during perioperative procedures. Studies are needed to confirm the previous findings and to add new knowledge to this area in Asian population. Our previous study assessed the use of epinephrine in managing patients with DIA through the analysis of the Beijing Pharmacovigilance Database (BPD) [10], and the present study is an extension of this project to provide a detailed analysis of the reported DIA cases.

\section{Aim of the study}

The objective of this study was to contribute to a better understanding of DIAs in Beijing, China, based on anaphylaxis case reports by the BPD over a decade period. The following information was extracted and analyzed: causative drugs, clinical features and severities of DIA cases.

\section{Ethics approval}

This study was considered to be exempt from further review by the Institutional Review Board, Peking University Third Hospital. Patient informed consent was not required because this was a retrospective study using only de-identified data.

\section{Method}

Using a structured database inquiry, extraction, and case adjudication methodology as reported in our previous study [10], we performed a detailed analysis on DIA cases. The cases were reported to the BPD from January 1, 2004 to December 31, 2014.

In contrast with our previous study [10], anaphylaxisinducing drugs were classified into various pharmacotherapeutic groups according to the Martindale-The Complete Drug Reference (37th edition) [11], World Health Organization (WHO) Model Formulary (2008) [12], and Chinese Pharmacopoeia (ChP) [13]. Within each group there were several subgroups. Previously, cases in which more than one drug was suspected were defined as "Associations" [10]. In this study, we further classified drugs under the "Associations" according to the original judgments of physicians when information is available. For example, a case was reported in which two drugs were administered to a patientAmbroxol injection and Lomefloxacin injection. Clinicians filing the report deemed that Lomefloxacin injection was more likely to induce anaphylaxis. Accordingly, we classified this case into "Antibiotics" instead of "Associations". For each DIA case, we assessed the case severity into three grades: grade 1 category was patients with only cutaneous involvement, grade 2 included patients with mild-to-moderate manifestations of anaphylaxis, and those with grade 3 reactions had severe presentations with cutaneous, gastrointestinal, and potentially life-threatening respiratory or cardiovascular signs and symptoms [14].

\section{Statistical analysis}

The statistical analysis was performed using the SPSS version 22 (SPSS Inc., IL, USA). Continuous variables were subjected to normality tests using the single sample 
Kolmogorov-Smirnov test, where data in accordance with normal distribution was expressed as mean \pm standard deviation, while those in accordance with the non-normal distribution was expressed as median (min, max), and the dichotomous variables were described as frequency (percentage).

\section{Results}

\section{Demographic and clinical characteristics}

A total of 9425 patients with drug-induced hypersensitivity reactions were identified from the BPD. After initial screening and adjudication, 1189 patients were ultimately included in our analysis [10]. Of these patients, the mean age was 47.6 years, $732(61.6 \%)$ were aged from 18 to 59 years. A total of 627 (52.7\%) were female patients (Table 1).

The majority of patients $(83.8 \%)$ experienced cardiovascular anaphylactic symptoms; the percentage of patients who developed mucocutaneous compromise, respiratory compromise, central nervous symptoms were 47.4, 55.4, and $50.1 \%$, respectively. Gastrointestinal anaphylactic symptoms occurred in $31.3 \%$ of the cases. Overall, 73 (6.1\%) of the patients were admitted to intensive care units (ICU), and 39

Table 1 Demographics, clinical characteristics and outcome of patients with drug-induced anaphylaxis

\begin{tabular}{lll}
\hline Variable & Value, no. $(\%)$ & $95 \%$ CI \\
\hline Demographics & & \\
Age & & \\
$\quad$ Mean-year & $47.6 \pm 20.1$ & $6.2-9.3$ \\
$\quad<18$ year & $91(7.7)$ & $58.8-64.4$ \\
$\quad 18-59$ year & $732(61.6)$ & $28.2-33.5$ \\
$\quad \geq 60$ year & $366(30.8)$ & $50.0-55.5$ \\
Female & $627(52.7)$ & $44.5-50.0$ \\
Male & $562(47.3)$ & $81.3-85.8$ \\
Organ system involvement & & $52.5-58.3$ \\
Cardiovascular & $996(83.8)$ & $47.4-53.1$ \\
Respiratory & $659(55.4)$ & $44.6-50.5$ \\
Central nervous system & $596(50.1)$ & $28.8-33.9$ \\
Mucocutaneous & $563(47.4)$ & \\
Gastrointestinal tract & $372(31.3)$ & $11.5-15.6$ \\
Severity of anaphylaxis & & $84.4-88.5$ \\
Mild to moderate & $160(13.5)$ & $4.9-7.8$ \\
Severe & $1029(86.5)$ & $2.3-4.3$ \\
Outcome & & \\
ICU admission & $73(6.1)$ & \\
Death & $39(3.3)$ & \\
\hline
\end{tabular}

$E D$ emergency department, $I C U$ intensive care unit
(3.3\%) patients died during their hospitalizations as a result of anaphylaxis.

\section{Drug triggers}

A total of 249 individual drugs were involved in the anaphylactic cases analyzed, classified into 23 pharmacotherapeutic groups and 53 subgroups. A total of $1145(96.3 \%)$ cases were attributed to single drugs, and 44 (3.7\%) were attributed to "Associations" (Table 2). While various drug triggers were reported, the main four general categories for DIAs were antibiotics (39.3\%), traditional Chinese medicines (TCM, $11.9 \%$ ), radiocontrast agents $(11.9 \%)$ and antineoplastic agents $(10.3 \%)$.

Antibiotics held the leading trigger medications of druginduced anaphylaxis $(467 / 1189,39.3 \%)$. Among the antibiotics, the top three sub-groups included beta-lactams (275/467, 58.9\%), fluoroquinolones (138/467, 29.6\%), and macrolides $(28 / 467,6.0 \%)$ (Table 3$)$. Within the beta lactams in particular, cephalosporins $(161 / 275,58.5 \%)$ were identified the most followed by beta-lactam/beta-lactamase inhibitors $(72 / 275,26.2 \%)$ and penicillins $(37 / 275,13.5 \%)$ (Table 3).

There were 141 DIA cases $(11.9 \%)$ induced by TCMs, most cases involving TCM injections (135/141, 95.7\%), with the remaining cases including oral or topical TCM formulations. A total of 36 different TCM injections were identified. These injections were mainly used for the treatment of cardiovascular and cerebrovascular disease, digestive system disease, respiratory system disease, and cancer. Ciwujia was the leading cause followed by Qingkailing, Houttuynia cordata, Shuxuening, Shuanghuanglian, Chuanhuning, Safflower and Yinxingdamo (Tables 2, 4). Other TCM injections (53 cases) were listed in Appendix 1 in the electronic supplementary materials.

Radiocontrast agents were reported 141 times, and the top three were contrast media used in X-ray $(113 / 141,80.1 \%)$, magnetic resonance imaging $(12 / 141,8.5 \%)$ and ophthalmic procedures (10/141, 7.1\%) (Tables 2, 4).

Among the DIA cases caused by antineoplastic drugs $(122 / 1189,10.3 \%)$, paclitaxel $(68 / 122,55.7 \%)$ and platinumbased antineoplastics (40/122, 32.8\%) were important contributors (Tables 2, 4). The remaining identified DIA cases (274) were listed in Table 2.

There were 149 anaphylaxis cases occurred during perioperative procedures, and the top three drug groups involved were antibiotics $(43.0 \%)$, radiocontrast agents $(14.8 \%)$ and plasma substitutes (9.4\%) (Appendix 2).

Most causative drugs were administered by the intravenous route $(86.4 \%)$, oral route $(5.4 \%)$, intramuscular route $(3.0 \%)$, subcutaneous route $(1.6 \%)$, and intra-arterial route $(1.4 \%)$. There were 12 cases of anaphylactic reactions occurred during intradermal tests. 
Table 2 Pharmacotherapeutic groups and subgroups involved of drug-induced anaphylaxis

\begin{tabular}{|c|c|c|}
\hline Drugs & Value, no. (\%) & $95 \% \mathrm{CI}$ \\
\hline Total & 1189 & \\
\hline Antibiotics & $467(39.3)$ & $36.7-42.1$ \\
\hline$\beta$-lactams & $275(23.1)$ & $21.0-25.5$ \\
\hline Fluoroquinolones & $138(11.6)$ & $9.8-13.4$ \\
\hline Macrolides & $28(2.4)$ & $1.6-3.3$ \\
\hline Other antibiotics $^{\mathrm{a}}$ & $26(2.2)$ & $1.3-3.0$ \\
\hline $\mathrm{TCM}$ & $141(11.9)$ & $10.2-14.3$ \\
\hline TCM (injection) & $135(11.4)$ & $9.5-13.2$ \\
\hline TCM (oral) & $5(0.4)$ & $0.1-0.8$ \\
\hline TCM (topical) & $1(0.1)$ & $0.0-0.3$ \\
\hline Radiocontrast agents & $141(11.9)$ & $10.2-14.3$ \\
\hline X-ray contrast media, iodinated & $113(9.5)$ & $7.8-11.3$ \\
\hline MRI contrast media & $12(1.0)$ & $0.5-1.6$ \\
\hline Ophthalmic medicines & $10(0.8)$ & $0.3-1.4$ \\
\hline Ultrasound contrast agents & $4(0.3)$ & $0.1-0.8$ \\
\hline Other $^{b}$ & $2(0.2)$ & $0.0-0.4$ \\
\hline Antineoplastics & $122(10.3)$ & $8.8-12.4$ \\
\hline Taxanes & $73(6.1)$ & $4.9-7.6$ \\
\hline Platinum compounds & $40(3.4)$ & $2.4-4.5$ \\
\hline Cytotoxic antibiotics & $5(0.4)$ & $0.1-0.8$ \\
\hline Alkylating agents & $2(0.2)$ & $0.0-0.4$ \\
\hline Teniposide & $1(0.1)$ & $0.0-0.3$ \\
\hline Asparaginase & $1(0.1)$ & $0.0-0.3$ \\
\hline Blood products, Biologics and plasma substitutes & $74(6.2)$ & $4.9-7.7$ \\
\hline Blood products and Biologics & $37(3.1)$ & $2.2-4.2$ \\
\hline Plasma substitutes & $25(2.1)$ & $1.3-3.0$ \\
\hline Monoclonal antibodies & $12(1.0)$ & $0.5-1.6$ \\
\hline Anesthetics & $25(2.1)$ & $1.3-3.0$ \\
\hline Local anesthetics & $10(0.8)$ & $0.3-1.4$ \\
\hline NMBAs & $12(1.0)$ & $0.5-1.6$ \\
\hline General anesthetics & $3(0.3)$ & $0.0-0.6$ \\
\hline Vaccines, immunoglobulins and antiserums & $23(1.9)$ & $1.2-2.8$ \\
\hline Immunoglobulins and antiserums & $19(1.6)$ & $0.9-2.4$ \\
\hline Vaccines & $4(0.3)$ & $0.1-0.7$ \\
\hline Nutrition and vitamins & $22(1.9)$ & $1.1-2.7$ \\
\hline Vitamins and minerals & $16(1.3)$ & $0.8-2.1$ \\
\hline Amino acids and fat emulsions & $6(0.5)$ & $0.2-0.9$ \\
\hline Immune-modulators & $21(1.8)$ & $1.0-2.5$ \\
\hline Immunostimulants & $16(1.3)$ & $0.8-1.9$ \\
\hline Immunosuppressants & $5(0.4)$ & $0.1-0.8$ \\
\hline Blood system medications & $20(1.7)$ & $0.9-2.4$ \\
\hline Antifibrinolytic agents and hemostatics & $11(0.9)$ & $0.4-1.5$ \\
\hline Iron (injection) & $6(0.5)$ & $0.2-1.0$ \\
\hline Anticoagulants & $2(0.2)$ & $0.0-0.4$ \\
\hline Antiplatelet agents & $1(0.1)$ & $0.0-0.3$ \\
\hline Analgesics, anti-inflammatory drugs and antipyretics & $17(1.4)$ & $0.8-2.2$ \\
\hline NSAIDs & $11(0.9)$ & $0.4-1.6$ \\
\hline Opioids & $5(0.4)$ & $0.1-0.8$ \\
\hline Paracetamol & $1(0.1)$ & $0.0-0.3$ \\
\hline Hormones, other endocrine medicines & $16(1.3)$ & $0.8-2.1$ \\
\hline Corticosteroids & $11(0.9)$ & $0.4-1.5$ \\
\hline
\end{tabular}


Table 2 (continued)

\begin{tabular}{|c|c|c|}
\hline Drugs & Value, no. $(\%)$ & $95 \% \mathrm{CI}$ \\
\hline Hypothalamic and pituitary hormones & $4(0.3)$ & $0.1-0.7$ \\
\hline Insulins & $1(0.1)$ & $0.0-0.3$ \\
\hline Prostaglandins & $10(0.8)$ & $0.3-1.4$ \\
\hline Cardiovascular medications & $11(0.9)$ & $0.4-1.5$ \\
\hline Vasodilators & $5(0.4)$ & $0.1-0.8$ \\
\hline Antiarrhythmics & $3(0.3)$ & $0.0-0.6$ \\
\hline $\mathrm{ACEI} / \mathrm{BB}$ & $2(0.2)$ & $0.0-0.4$ \\
\hline Statins & $1(0.1)$ & $0.0-0.3$ \\
\hline Gastrointestinal medicines & $8(0.7)$ & $0.3-1.1$ \\
\hline $\mathrm{H} 2$ receptor blockers & $3(0.3)$ & $0.0-0.6$ \\
\hline Laxatives & $2(0.2)$ & $0.0-0.4$ \\
\hline PPI & $1(0.1)$ & $0.0-0.3$ \\
\hline Antacids & $1(0.1)$ & $0.0-0.3$ \\
\hline Antiemetics & $1(0.1)$ & $0.0-0.3$ \\
\hline Antidotes & $7(0.6)$ & $0.3-1.1$ \\
\hline Antivirals & $5(0.4)$ & $0.1-0.8$ \\
\hline Antifungals & $3(0.3)$ & $0.0-0.6$ \\
\hline Psychotherapeutic medicines & $3(0.3)$ & $0.0-0.6$ \\
\hline Antidementia medicines & $2(0.2)$ & $0.0-0.4$ \\
\hline Antipsychotic medications & $1(0.1)$ & $0.0-0.3$ \\
\hline Respiratory medications & $3(0.3)$ & $0.0-0.6$ \\
\hline Expectorants & $2(0.2)$ & $0.0-0.4$ \\
\hline Bronchodilators & $1(0.1)$ & $0.0-0.3$ \\
\hline Calcium regulating drugs & $1(0.1)$ & $0.0-0.3$ \\
\hline Associations $^{\mathrm{c}}$ & $44(3.7)$ & $2.7-4.9$ \\
\hline Others $^{\mathrm{d}}$ & $5(0.4)$ & $0.1-0.8$ \\
\hline
\end{tabular}

TCM traditional Chinese medicine, NSAIDs non-steroidal anti-inflammatory drugs, MRI magnetic resonance imaging, $N M B A s$ neuromuscular blocking agents, $A C E I$ angiotensin converting enzyme inhibitor; $B B$ beta-blocker, PPI proton pump inhibitor

${ }^{a}$ Other antibiotics included aminoglycosides, clindamycin, vancomycin, and metronidazole

${ }^{\mathrm{b}}$ Other radiocontrast agent was indocyanine green injection

${ }^{\mathrm{c}}$ Associations were defined as those cases in which more than one medication was suspected to cause the anaphylaxis. Details of anaphylaxis induced by associations were listed in Appendix 3

d“"Others” category included monosialotetrahexosylganglioside sodium for injection, sodium deoxyribonucleotide injection, cerebroprotein hydrolysate for injection, and coenzyme A for injection

Of the 44 DIA cases caused by the association of two or more drugs, $14(31.8 \%)$ anaphylaxis cases involved one or more TCM combined with one or more other non-TCM, 12 (27.3\%) occurred during general anesthesia, and $21(47.7 \%)$ included antibiotics (Appendix 3).

\section{Severity and anaphylaxis-related deaths}

Among the 1189 DIA cases, 1029 (86.5\%) were considered as severe (grade 3 ) and 39 (3.3\%) were fatal. Mortality was due mainly to the following top four medication groups: antibiotics $(13 / 39,33.3 \%)$, radiocontrast agents (12/39, $30.8 \%)$, antineoplastic agents $(4 / 39,10.3 \%)$, TCM injections $(3 / 39,7.7 \%)$. The detailed information was listed in Table 5.

\section{Discussion}

To our knowledge, this is the first analysis of drug-induced anaphylaxis in the hospital setting in China. Using the Beijing Pharmacovigilance Database, our study shows that there were 1189 DIA cases in clinical settings over a decade in Beijing, China, accounting for $12.6 \%$ of all ADE reports collected by the BPD. The percentage of DIA in Beijing, China is higher than the percentage of DIA in Portugal (5-7\%) spanning over a decade [8]. Patients aged between 18 and 59 years $(61.6 \%)$ had the highest frequency of DIA among all three age groups, which is consistent with a recent study in China by Jiang et al. [15]. Similar to most studies [8, 9, 16], females had a higher frequency of anaphylaxis compared to males in our analysis. A high proportion of patients 
Table 3 Drugs of antibiotic-induced anaphylaxis

\begin{tabular}{|c|c|c|}
\hline Antibiotics & ATC-codes & $\begin{array}{l}\text { All Patients } \\
(\mathrm{n}=467) \text { Value, } \\
\text { no. }(\%)\end{array}$ \\
\hline$\beta$-Lactam antibiotics & & $275(58.9)$ \\
\hline Cephalosporins & & $161(34.5)$ \\
\hline First-generation & J01DB & $13(2.8)$ \\
\hline cefradine & J01DB09 & $4(0.9)$ \\
\hline cefalexin & J01DB01 & $4(0.9)$ \\
\hline cefazolin & J01DB04 & $3(0.6)$ \\
\hline cefadroxil & J01DB05 & $1(0.2)$ \\
\hline cefathiamidine & NA & $1(0.2)$ \\
\hline Second-generation & J01DC & $86(18.4)$ \\
\hline cefuroxime & J01DC02 & $54(11.6)$ \\
\hline cefmetazole & J01DC09 & $18(3.9)$ \\
\hline cefoxitin & J01DC01 & $6(1.3)$ \\
\hline cefotiam & J01DC07 & $3(0.6)$ \\
\hline cefminox & J01DC12 & $2(0.4)$ \\
\hline cefamandole & J01DC03 & $2(0.4)$ \\
\hline cefaclor & J01DC04 & $1(0.2)$ \\
\hline Third-generation & J01DD & $58(12.4)$ \\
\hline ceftriaxone & J01DD04 & $35(7.5)$ \\
\hline ceftazidime & J01DD02 & $10(2.1)$ \\
\hline ceftizoxime & J01DD07 & $6(1.3)$ \\
\hline cefoperazone & J01DD12 & $4(0.9)$ \\
\hline cefotaxime & J01DD01 & $2(0.4)$ \\
\hline cefdinir & J01DD15 & $1(0.2)$ \\
\hline Fourth-generation & J01DE & $4(0.9)$ \\
\hline cefepime & J01DE01 & $4(0.9)$ \\
\hline$\beta$-lactam $+\beta$-lactamase inhibitors & & $72(15.4)$ \\
\hline cefoperazone + sulbactam & J01DD62 & $45(9.6)$ \\
\hline piperacillin + sulbactam & J01CR05 & $12(2.6)$ \\
\hline piperacillin + tazobactam & J01CR05 & $5(1.1)$ \\
\hline ampicillin + sulbactam & J01CA51 & $5(1.1)$ \\
\hline amoxicillin + clavulanic acid & J01CR02 & $3(0.6)$ \\
\hline imipenem + cilastatin & J01DH51 & $1(0.2)$ \\
\hline amoxicillin + sulbactam & J01CR02 & $1(0.2)$ \\
\hline Penicillins & $\mathrm{J} 01 \mathrm{C}$ & 37 (7.9) \\
\hline benzylpenicillin & J01CE01 & $23(4.9)$ \\
\hline $\begin{array}{l}\text { Penicillins with extended spec- } \\
\text { trum (aminopenicillins) }\end{array}$ & J01CA & $14(3.0)$ \\
\hline azlocillin & J01CA09 & $5(1.1)$ \\
\hline amoxicillin & J01CA04 & $5(1.1)$ \\
\hline mezlocillin & J01CA10 & $4(0.9)$ \\
\hline Others & & $5(1.1)$ \\
\hline aztreonam & J01DF01 & $3(0.6)$ \\
\hline latamoxef & J01DD06 & $2(0.4)$ \\
\hline Fluoroquinolones & J01MA & $138(29.6)$ \\
\hline levofloxacin & J01MA12 & $82(17.6)$ \\
\hline moxifloxacin & J01MA14 & $23(4.9)$ \\
\hline gatifloxacin & J01MA16 & $14(3.0)$ \\
\hline pefloxacin & J01MA03 & $5(1.1)$ \\
\hline
\end{tabular}

Table 3 (continued)

\begin{tabular}{llc}
\hline Antibiotics & ATC-codes & $\begin{array}{l}\text { All Patients } \\
(\mathrm{n}=467) \text { Value, } \\
\text { no. }(\%)\end{array}$ \\
\hline ofloxacin & J01MA01 & $4(0.9)$ \\
fleroxacin & J01MA08 & $4(0.9)$ \\
ciprofloxacin & J01MA02 & $4(0.9)$ \\
lomefloxacin & J01MA07 & $2(0.4)$ \\
Macrolides & J01FA & $28(6.0)$ \\
azithromycin & J01FA10 & $28(6.0)$ \\
clindamycin & J01FF01 & $14(3.0)$ \\
Aminoglycosides & J01G & $8(1.7)$ \\
etimicin & NA & $7(1.5)$ \\
gentamicin & J01GB03 & $1(0.2)$ \\
vancomycin & J01XA01 & $3(0.6)$ \\
metronidazole & J01XD01 & $1(0.2)$ \\
\hline
\end{tabular}

ATC anatomical therapeutic chemical, $N A$ not available

developed cardiovascular symptoms (83.8\%), and the mortality rate of $3.3 \%$ is comparable to previous studies $[17,18]$.

\section{Antibiotic-induced anaphylaxis}

Of all DIAs reported in BPD and included within our analysis, antibiotics attributed to the most common cause of DIA (39.3\%), comparable to the incidence of antibiotic-induced anaphylaxis within the U.S. (40.5\%) [6]. However, antibiotic-induced anaphylaxis occurred more frequently than reports from Korea (10.5\%) [7] and Portugal (16.7\%) [8]. In agreement with previous reports, anaphylaxis from $\beta$-lactam antibiotics (58.9\%) were reported more frequently than non$\beta$-lactam antibiotics [19]. Cephalosporins accounted for the majority of $\beta$-lactam antibiotic-related anaphylaxis followed by beta-lactam/beta-lactamase inhibitors and penicillins. This may be explained partly by the greater use of cephalosporins in Chinese hospitals [20]. The likelihood of anaphylaxis from penicillins can be assessed by skin tests [19]. However, routine intradermal skin testing of a cephalosporin may not be useful for predicting an immediate hypersensitivity because of the extremely low test sensitivity, which was confirmed by a recent retrospective study conducted by Yoon et al. [21]. Fluoroquinolone antibiotics also accounted for a high proportion of all 1189 anaphylaxis cases (11.6\%), which is consistent with the frequency $(11.7 \%)$ found by Faria et al. [16]. The high frequency of anaphylaxis from fluoroquinolones maybe due to the overuse of fluoroquinolone antibiotics in China [20], and the hypersensitivity to quinolones is less likely to be determined from skin testing [2].

Given that antibiotics are the most common trigger for anaphylaxis, the importance of inquiring and documenting patient's medication allergic history cannot be 
Table 4 Drugs of radiocontrast-induced anaphylaxis, TCM injectioninduced anaphylaxis, and antineoplastics-induced anaphylaxis

\begin{tabular}{|c|c|c|}
\hline Drugs & ATC-codes & Value, no. (\%) \\
\hline \multicolumn{3}{|l|}{ Radiocontrast agents $(\mathrm{n}=141)$} \\
\hline iopromide & V08AB05 & $52(36.9)$ \\
\hline iohexol & V08AB02 & $26(18.4)$ \\
\hline iopamidol & V08AB04 & $16(11.3)$ \\
\hline fluorescein sodium & NA & $10(7.1)$ \\
\hline gadopentetic acid (gadopentetate dimeglumine) & V08CA01 & $9(6.4)$ \\
\hline ioversol & V08AB07 & $7(5.0)$ \\
\hline iobitridol & V08AB11 & $5(3.5)$ \\
\hline iodixanol & V08AB09 & $5(3.5)$ \\
\hline sulfur hexafluoride & V08DA05 & $4(2.8)$ \\
\hline diatrizoic acid (meglumine diatrizoate) & V08AA01 & $2(1.4)$ \\
\hline indocyanine green & NA & $2(1.4)$ \\
\hline gadobenic acid (gadobenate dimeglumine) & V08CA08 & $1(0.7)$ \\
\hline gadodiamide & V08CA03 & $1(0.7)$ \\
\hline gadoteric acid (gadoterate meglumine) & V08CA02 & $1(0.7)$ \\
\hline \multicolumn{3}{|l|}{ TCM injections $(\mathrm{n}=135)$} \\
\hline Ciwujia & NA & $21(15.6)$ \\
\hline Qingkailing & NA & $16(11.9)$ \\
\hline Houttuynia cordata & NA & $12(8.9)$ \\
\hline Shuxuening & NA & $11(8.1)$ \\
\hline Shuanghuanglian & NA & $6(4.4)$ \\
\hline Chuanhuning & NA & $6(4.4)$ \\
\hline Safflower & NA & $5(3.7)$ \\
\hline Yinxingdamo & NA & $5(3.7)$ \\
\hline Others $^{\mathrm{a}}$ & NA & $53(39.3)$ \\
\hline \multicolumn{3}{|l|}{ Antineoplastics $(\mathrm{n}=122)$} \\
\hline paclitaxel & L01CD01 & $68(55.7)$ \\
\hline oxaliplatin & L01XA03 & $18(14.8)$ \\
\hline carboplatin & L01XA02 & $13(10.7)$ \\
\hline cisplatin & L01XA01 & $7(5.7)$ \\
\hline docetaxel & L01CD02 & $5(4.1)$ \\
\hline cyclophosphamide & L01AA01 & $2(1.6)$ \\
\hline doxorubicin & L01DB01 & $2(1.6)$ \\
\hline nedaplatin & NA & $2(1.6)$ \\
\hline topotecan & L01XX17 & $1(0.8)$ \\
\hline epirubicin & L01DB03 & $1(0.8)$ \\
\hline bleomycin A5 & NA & $1(0.8)$ \\
\hline mitomycin & L01DC03 & $1(0.8)$ \\
\hline asparaginase & L01XX02 & $1(0.8)$ \\
\hline
\end{tabular}

TCM traditional Chinese medicine, ATC anatomical therapeutic chemical, NA not available

${ }^{a}$ Only the top 8 common TCMs were listed; detailed information on the Others category was presented in Appendix 1 in the electronic supplementary materials underestimated. This is the area pharmacists could contribute to patient safety. Clinical symptoms and signs related to anaphylaxis should be closely monitored when antibiotics, especially cephalosporins and fluoroquinolones, are administered to patients in the hospital setting.

\section{TCM-induced anaphylaxis}

The use of TCM was the second most common drug trigger in our analysis, and almost all (95.7\%) TCM-related anaphylactic cases were from injectable TCM formulations. 
Table 5 Description of the anaphylaxis-related deaths

\begin{tabular}{|c|c|c|}
\hline Variable & ATC-codes & $\begin{array}{l}\text { All patients } \\
(\mathrm{n}=39) \text { value, } \\
\text { no. }(\%)\end{array}$ \\
\hline \multicolumn{3}{|l|}{ Age } \\
\hline Mean-year & & $53.8 \pm 19.1$ \\
\hline$<18$ year & & $1(2.6)$ \\
\hline $18-59$ year & & $20(51.3)$ \\
\hline$\geq 60$ year & & $18(46.1)$ \\
\hline Female & & $14(35.9)$ \\
\hline Antibiotics & & $13(33.3)$ \\
\hline cefuroxime & J01DC02 & $5(12.8)$ \\
\hline levofloxacin & J01MA12 & $3(7.7)$ \\
\hline ceftriaxone & J01DD04 & $1(2.6)$ \\
\hline ceftizoxime & J01DD07 & $1(2.6)$ \\
\hline cefepime & J01DE01 & $1(2.6)$ \\
\hline piperacillin + sulbactam & J01CR05 & $1(2.6)$ \\
\hline clindamycin & J01FF01 & $1(2.6)$ \\
\hline Radiocontrast agents & & $12(30.8)$ \\
\hline ioversol & V08AB07 & $4(10.3)$ \\
\hline iohexol & V08AB02 & $3(7.7)$ \\
\hline iopromide & V08AB05 & $2(5.1)$ \\
\hline iopamidol & V08AB04 & $1(2.6)$ \\
\hline $\begin{array}{l}\text { gadopentetic acid (gado- } \\
\text { pentetate dimeglumine) }\end{array}$ & V08CA01 & $1(2.6)$ \\
\hline fluorescein sodium & NA & $1(2.6)$ \\
\hline Antineoplastics & & $4(10.3)$ \\
\hline paclitaxel & L01CD01 & $2(5.1)$ \\
\hline oxaliplatin & L01XA03 & $1(2.6)$ \\
\hline asparaginase & L01XX02 & $1(2.6)$ \\
\hline TCM injections & & $3(7.7)$ \\
\hline Houttuynia cordata & NA & $2(5.1)$ \\
\hline Chuanhuning & NA & $1(2.6)$ \\
\hline plasma substitutes & & $2(5.1)$ \\
\hline hydroxyethyl starch & B05AA07 & $1(2.6)$ \\
\hline dextran & B05AA05 & $1(2.6)$ \\
\hline Vaccine & J07BB03 & $1(2.6)$ \\
\hline protamine & V03AB14 & $1(2.6)$ \\
\hline articaine & N01BB08 & $1(2.6)$ \\
\hline doxofylline & R03DA11 & $1(2.6)$ \\
\hline Associations $^{\mathrm{a}}$ & & $1(2.6)$ \\
\hline
\end{tabular}

TCM traditional Chinese medicine, ATC anatomical therapeutic chemical, NA not available

${ }^{a}$ Associations were Moxifloxacin hydrochloride and sodium chloride injection, and Ambroxol hydrochloride for injection

The result is similar to a study reported by Jiang et al. [15]. TCM is unique to the Chinese population and therefore the DIAs associated with TCM is unique to that population as a result. Along with extensive indications for TCM, there is greater use of TCM injections compared to other countries. In our review, 36 different TCM injections were identified resulting in $11.4 \%$ of the anaphylaxis cases, among which three cases were fatal. The four most common triggers were injections of Ciwujia, Qingkailing, Houttuynia cordata and Shuxuening. The results were similar to those of Jiang et al., who found the most common triggers were injections of Qingkailing, Shuanghuanglian and Houttuynia cordata [15].

TCM injection is extracted from Chinese herbs, which may contain one or several active ingredients. The high frequency of anaphylactic cases related to TCM injections may be explained as follows: (1) the components of a TCM injection are relatively complex and most formulations have not been thoroughly analyzed and identified; (2) a TCM injection also contains various additives, such as pigment, tannin, starch and protein, and these additives may trigger the body's immune system and cause anaphylaxis [22]; (3) quality control of TCM injections is relatively difficult due to the formulation complexity, and impurities may cause anaphylaxis as well; and (4) drug interactions between TCM injections and other medications should also be considered. Of the 135 patients who suffered anaphylaxis induced by TCM injections, patients' age ranged from 4 to 90 years: children under 18 years (5\%), adults between 18 and 59 years (58.6\%), and adults over 60 years (36.4\%). This is consistent with a previous study reporting that patient of any age can suffer anaphylaxis induced by TCM injections [22]. Therefore, caution should be exercised before considering the use of TCM and all patients regardless of age should be closely monitored during TCM administration.

\section{Radiocontrast-induced anaphylaxis}

Our study found that radiocontrast agents were the third most common cause $(11.9 \%)$ of DIA, coinciding with the frequency of previous studies in Korea (12.0\%) [7]. However DIA through radiocontrast agents was reported less frequently than that in the United States (30.4\%) [6]. Of these radiocontrast-induced anaphylaxis cases, the majority $(80.1 \%)$ were caused by iodine-based contrast agents. The number of anaphylaxis cases induced by iopromide $(36.9 \%)$ were greater than any other non-ionic iodinated contrast agents, which is similar to the results from a previous study [23]. A recent retrospective study from Korea indicated that among the anaphylactic patients, iopromide was associated with more severe anaphylaxis with hypotension [24].

Patients with allergies, asthma, renal insufficiency, anxiety, significant cardiac disease and other miscellaneous risk factors may be at an increased risk for anaphylactoid contrast reactions [25]. Patient's medical history should be collected and the risk of contrast media induced anaphylactic reactions should be assessment before contrast media administration. More importantly, appropriate resuscitative equipment should be available to treat anaphylactic reactions promptly. 
Premedications such as corticosteroids should also be used for pretreatment of "at-risk" patients who require a contrastenhanced examination [25].

\section{Antineoplastics-induced anaphylaxis}

Antineoplastic agents were another frequent cause of DIA in the current study, consistent with those reported in literature $[6,8]$. This may be related to the increasing chemotherapy use with ever increasing cancer prevalence in Beijing, China [26]. Paclitaxel accounted for $55.7 \%$ of antineoplastics-induced anaphylaxis, and the high frequency may be related to the solubilizer, polyoxyethylene castor oil, which can induce anaphylaxis [27]. We also found that platinum-based agents accounted for $32.8 \%$ of all antineoplastics-induced anaphylaxis cases. Among these agents, oxaliplatin was a major trigger accounting for $1.5 \%$ of all 1189 DIA cases, and this finding is consistent with a published study [28].

Although a recent study from Nonna et al. found that for patients with carboplatin induced hypersensitivity reaction, the use of oxaliplatin maybe a safer alternative [29], patients should be carefully monitored for signs and symptoms of anaphylaxis with any platinum-based chemotherapeutic agent.

\section{Limitations}

Our retrospective analysis was based on self-reported cases by health care professionals from the BPD, and therefore our study has the following limitations: (1) lack of the frequency of causative drug use as we could not obtain either prescription or reimbursement data; (2) we could not assess the prevalence of DIA in the region studied as we do not have the information of the total patient base; (3) potential reporting bias may exist: majority of reported cases were hospitalized patients in the non-ED setting, and only severe anaphylactic cases may have been reported; (4) we may not have included all DIA cases in the BPD: cases missed if clinicians did not report using the terms related to allergy or anaphylaxis or hypersensitivity (e.g. a patient with wheeze, vomiting, bronchospasm but was not described as "allergy" by clinicians when reporting to the BPD). In addition, some reported cases were not included due to insufficient information. Despite these limitations, the method we have taken should be robust against a range of potential biases: rigorous inclusion/exclusion criteria were utilized and all potential anaphylaxis cases were adjudicated by trained physician/allergists; and only patients with confirmed anaphylaxis and complete data record were included in the analysis.

\section{Conclusion}

This first detailed analysis of DIA case reports from 2004 to 2014 in Chinese patients provides valuable information to clinicians. Antibiotics, TCM, radiocontrast media and antineoplastic agents are the most common causes of DIA cases. The majority of DIA cases are considered to be severe with a high mortality rate of $3.3 \%$. Pharmacists should be working closely with prescribers to assess each patient's risks of developing anaphylaxis when drug therapy is involved, and to provide prompt treatment and resuscitations to reduce the morbidity and mortality when anaphylaxis occurs.

Acknowledgements The authors would like to thank the Beijing Pharmacovigilance Database for providing data.

Funding This research is partially supported by the Research Grant 892FY60221022 from School of Pharmaceutical Sciences, Peking University.

Conflicts of interest The authors have no conflicts of interest to declare.

Open Access This article is distributed under the terms of the Creative Commons Attribution 4.0 International License (http://creativecommons.org/licenses/by/4.0/), which permits unrestricted use, distribution, and reproduction in any medium, provided you give appropriate credit to the original author(s) and the source, provide a link to the Creative Commons license, and indicate if changes were made.

\section{References}

1. Muraro A, Roberts G, Worm M, Bilo MB, Brockow K, Fernandez Rivas M, et al. Anaphylaxis: guidelines from the European academy of allergy and clinical immunology. Allergy. 2014;69(8):1026-45.

2. Simons FE, Ebisawa M, Sanchez-Borges M, Thong BY, Worm M, Tanno LK, et al. 2015 update of the evidence base: World Allergy Organization anaphylaxis guidelines. World Allergy Organ J. 2015;8(1):32.

3. Panesar SS, Javad S, de Silva D, Nwaru BI, Hickstein L, Muraro A, et al. The epidemiology of anaphylaxis in Europe: a systematic review. Allergy. 2013;68(11):1353-61.

4. Cianferoni A, Novembre E, Mugnaini L, Lombardi E, Bernardini $\mathrm{R}$, Pucci N, et al. Clinical features of acute anaphylaxis in patients admitted to a university hospital: an 11-year retrospective review (1985-1996). Ann Allergy Asthma Immunol. 2001;87(1):27-32.

5. Renaudin JM, Beaudouin E, Ponvert C, Demoly P, Moneret-Vautrin DA. Severe drug-induced anaphylaxis: analysis of 333 cases recorded by the Allergy Vigilance Network from 2002 to 2010. Allergy. 2013;68(7):929-37.

6. Jerschow E, Lin RY, Scaperotti MM, McGinn AP. Fatal anaphylaxis in the United States, 1999-2010: temporal patterns and demographic associations. J Allergy Clin Immunol. 2014;134(6):1318-28.e7.

7. Ye YM, Kim MK, Kang HR, Kim TB, Sohn SW, Koh YI, et al. Predictors of the severity and serious outcomes of anaphylaxis in korean adults: a multicenter retrospective case study. Allergy Asthma Immunol Res. 2015;7(1):22-9. 
8. Ribeiro-Vaz I, Marques J, Demoly P, Polonia J, Gomes ER. Drug-induced anaphylaxis: a decade review of reporting to the Portuguese Pharmacovigilance Authority. Eur J Clin Pharmacol. 2013;69(3):673-81.

9. Sole D, Ivancevich JC, Borges MS, Coelho MA, Rosario NA, Ardusso LR, et al. Anaphylaxis in Latin America: a report of the online Latin American survey on anaphylaxis (OLASA). Clinics (Sao Paulo). 2011;66(6):943-7.

10. Wang T, Ma X, Xing Y, Sun S, Zhang H, Sturmer T, et al. Use of epinephrine in patients with drug-induced anaphylaxis: an analysis of the Beijing Pharmacovigilance Database. Int Arch Allergy Immunol. 2017;173(1):51-60.

11. Sweetman S. Martindale: the complete drug reference, 37 th ed. London: Pharmaceutical Press; 2011. ISBN 9780853699330.

12. World Health Organization. WHO model formulary 2008. ISBN 9789241547659. http://apps.who.int/medicinedocs/en/m/abstract/ Js16879e/. Accessed 8 May 2017.

13. Chinese Pharmacopoeia Commission. Chinese pharmacopoeia (Volume I). Beijing: China Medical Science Press; 2015. ISBN 9787506775397

14. Brown AF, McKinnon D, Chu K. Emergency department anaphylaxis: a review of 142 patients in a single year. J Allergy Clin Immunol. 2001;108(5):861-6.

15. Jiang N, Yin J, Wen L, Li H. Characteristics of anaphylaxis in 907 Chinese patients referred to a tertiary allergy center: a retrospective study of 1,952 episodes. Allergy Asthma Immunol Res. 2016;8(4):353-61.

16. Faria E, Rodrigues-Cernadas J, Gaspar A, Botelho C, Castro E, Lopes A, et al. Drug-induced anaphylaxis survey in Portuguese Allergy Departments. J Investig Allergol Clin Immunol. 2014;24(1):40-8.

17. Baldo BA, Pham NH. Classification and descriptions of allergic reactions to drugs. Drug allergy: Clinical aspects, diagnosis, mechanisms, structure-activity relationships. New York, NY: Springer; 2013. p. 15-35.

18. Fisher M, Baldo BA. Anaphylaxis during anaesthesia: current aspects of diagnosis and prevention. Eur $\mathbf{J}$ Anaesthesiol. 1994;11(4):263-84.

19. Lieberman P, Nicklas RA, Oppenheimer J, Kemp SF, Lang DM, Bernstein DI, et al. The diagnosis and management of anaphylaxis practice parameter: 2010 update. J Allergy Clin Immunol. 2010;126(3):477-80.e1-42.

20. Wang J, Wang P, Wang X, Zheng Y, Xiao Y. Use and prescription of antibiotics in primary health care settings in China. JAMA Intern Med. 2014;174(12):1914-20.

21. Yoon SY, Park SY, Kim S, Lee T, Lee YS, Kwon HS, et al. Validation of the cephalosporin intradermal skin test for predicting immediate hypersensitivity: a prospective study with drug challenge. Allergy. 2013;68(7):938-44.

22. Guo YJ, Wang DW, Meng L, Wang YQ. Analysis of anaphylactic shock caused by 17 types of traditional Chinese medicine injections used to treat cardiovascular and cerebrovascular diseases. Biomed Res Int. 2015;2015:420607.

23. Kim SR, Lee JH, Park KH, Park HJ, Park JW. Varied incidence of immediate adverse reactions to low-osmolar non-ionic iodide radiocontrast media used in computed tomography. Clin Exp Allergy. 2016;47(1):106-12.

24. Kim MH, Lee SY, Lee SE, Yang MS, Jung JW, Park CM, et al. Anaphylaxis to iodinated contrast media: clinical characteristics related with development of anaphylactic shock. PLoS ONE. 2014;9(6):e100154.

25. American College of Radiology. ACR manual on contrast media (v10.3). ISBN 9781559030120. https://www.acr.org/QualitySafety/Resources/Contrast-Manual. Accessed 8 May 2017.

26. Chen W, Zheng R, Baade PD, Zhang S, Zeng H, Bray F, et al. Cancer statistics in China, 2015. CA Cancer J Clin. 2016;66(2):115-32.

27. Szebeni J. Complement activation-related pseudoallergy: a new class of drug-induced acute immune toxicity. Toxicology. 2005;216(2-3):106-21.

28. Wang JH, King TM, Chang MC, Hsu CW. Oxaliplatin-induced severe anaphylactic reactions in metastatic colorectal cancer: case series analysis. World J Gastroenterol. 2012;18(38):5427-33.

29. Kolomeyevskaya NV, Lele SB, Miller A, Riebandt GC, Blum $\mathrm{BL}$, Odunsi $\mathrm{KO}$, et al. Oxaliplatin is a safe alternative option for patients with recurrent gynecologic cancers after hypersensitivity reaction to Carboplatin. Int J Gynecol Cancer. 2015;25(1):42-8. 